\title{
Theoretical investigation of the structural, elastic, electronic, and dielectric properties of alkali-metal-based bismuth ternary chalcogenides
}

\author{
Syam Kumar R $\odot,{ }^{1, *}$ Akinlolu Akande $\odot,{ }^{1}$ Fedwa El-Mellouhi $\odot,{ }^{2}$ Heesoo Park $\odot,{ }^{2}$ and Stefano Sanvito $\odot^{3}$ \\ ${ }^{1}$ Department of Health and Nutritional Science, Institute of Technology Sligo, Ash Lane, Co. Sligo, F91 YW50, Ireland \\ ${ }^{2}$ Qatar Environment and Energy Research Institute, Hamad Bin Khalifa University, P.O. Box 34110, Doha, Qatar \\ ${ }^{3}$ School of Physics, AMBER and CRANN Institute, Trinity College, Dublin 2, Ireland
}

(Received 18 December 2019; accepted 11 May 2020; published 10 July 2020)

\begin{abstract}
The past decade has witnessed the rapid introduction of organic-inorganic hybrid compounds in photovoltaic applications. Motivated by the strong demand for stable and nontoxic materials in this class, we report a theoretical study on the structural, elastic, electronic, thermodynamic and dielectric properties of alkali-metal-based bismuth ternary chalcogenides. In particular, we employ state-of-the-art density functional theory to explore the potential of $A \mathrm{Bi} X_{2}$ and $A \mathrm{Bi} X_{3}(A=\mathrm{Na}, \mathrm{K}$ and $X=\mathrm{O}, \mathrm{S})$ as light-absorbing media. All the compounds under investigation are found to be thermodynamically and mechanically stable, with a semiconductor band structure. The Kohn-Sham band gaps range between $0.80 \mathrm{eV}$ and $1.80 \mathrm{eV}$, when calculated with semilocal functionals, values that increase to $1.24-2.47 \mathrm{eV}$ with hybrid ones. Although all but $\mathrm{NaBiO}_{2}$ and $\mathrm{KBiO}_{2}$ are indirect band-gap semiconductors, the onset of the imaginary part of their dielectric functions, the optical gap, is only marginally larger than the quasiparticle gap. This is due to the generally flat nature of both the conduction and the valence bands. We then expect these compounds to absorb light in the upper part of the visible spectrum. In all cases the valence band is dominated by $\mathrm{O}-p$ and $\mathrm{S}-p$ orbitals and the conduction one by Bi- $p$, suggesting the possibility of excitons with low binding energy. The only exceptions are $\mathrm{NaBiO}_{2}$ and $\mathrm{KBiO}_{2}$ for which the $\mathrm{O}-p$ states dominate the density of states at both sides of the band gap.
\end{abstract}

DOI: 10.1103/PhysRevMaterials.4.075401

\section{INTRODUCTION}

The twenty first century has witnessed a rising demand for efficient alternative energy sources to substitute the rapidly depleting fossil fuel reserves and to provide greener and more sustainable solutions to the global energy demand. Solar power is commonly recognized to be the ultimate unlimited energy source, but its harvesting is not free of challenge. The simplest, oldest, and most commonly used solar photovoltaic (PV) device is the silicon solar cell. In fact, silicon in both its mono- and polycrystalline form is still the dominant material in PV technology and takes more than $90 \%$ of the market share [1]. Alternative materials platforms for PV, which have already enjoyed some commercial exploitation, include CdTe and $\mathrm{CuIn}_{x} \mathrm{Ga}_{1-x} \mathrm{Se}_{2}$ (CIGS) thin films [2,3]. All these PV media, unfortunately, share the need for relatively expensive high-temperature processing, which offsets the low cost of the associated raw materials. The second drawback of Si-based $\mathrm{PV}$ technology is that Si is generally brittle, so that it can be hardly integrated over the flexible substrates required by some mobile applications (e.g., wearable electronics). For these reasons, significant research has been dedicated to exploring materials solutions alternative to $\mathrm{Si}$. These include III-IV

\footnotetext{
*syamkumar.rameshkumar@mail.itsligo.ie

Published by the American Physical Society under the terms of the Creative Commons Attribution 4.0 International license. Further distribution of this work must maintain attribution to the author(s) and the published article's title, journal citation, and DOI.
}

semiconductors (in particular for multiple-junction tandem cells), dye-sensitized and organic compounds, GaAs-based structures, and intermediate-band-gap solar materials [4-8].

A materials family, which has recently attracted enormous attention, is that of the perovskites, in particular hybrid organic-inorganic perovskites (HOIPs). Discovered in the late 80's [9] and early 90's [10], HOIPs are generally easy to synthesize and show strong light properties [11]. Solar cells made of HOIPs have reached efficiencies exceeding $20 \%$ in less than a decade from their discovery [12] and are now considered among the top candidate materials for affordable PV technologies. Among their properties, these compounds are solution processable $[13,14]$ and they can be deposited cheaply by spin coating from solution and by co-evaporation [15-17]. $\mathrm{MAPbI}_{3}(\mathrm{MA}=$ methylammonium, $\mathrm{CH}_{3} \mathrm{NH}_{3}$ ) is the most celebrated member of this family, having an experimental band gap conveniently placed at the optimum value of the AM1.5G solar illumination spectrum [18] and presenting a high absorption coefficient. This feature allows one to fabricate films thinner than the carrier diffusion length for an optimal carrier-generation profile [7].

Unfortunately, there are also concerns around HOIPs. These are related to their stability in the air, in particular, their fragility against humidity, and the health issues arising from the presence of the toxic element $\mathrm{Pb}$ [19]. A number of strategies have been proposed to overcome these limitations. Some of them have an engineering nature, for instance, the attempt to encapsulate $\mathrm{MAPbI}_{3}$ within humidity-protecting layers, but most of the effort has concentrated on synthesising alternative compounds. The replacement of MA with other organic molecules generally tends to reduce the band gap and 
may further compromise the stability [20], while substituting $\mathrm{Pb}$ with $\mathrm{Sn}$ results in air-unstable compounds because of the ease of oxidation of the $\mathrm{Sn}^{2+}$ ion [13,21]. Mixed-cation [20] and double perovskites [22] have also been considered, although tuning their properties is a complex task. All these attempts suggest that surpassing the $\mathrm{PV}$ properties of $\mathrm{MAPbI}_{3}$ is by no means simple. Intriguingly, the unique characteristic of the $\mathrm{Pb}$-based HOIPs is the presence of the $\mathrm{Pb} 6 s^{2}$ lone pair that causes strong $\mathrm{Pb} 6 s-\mathrm{I} 5 p$ antibonding character of the valence band of the high-symmetry perovskite structure [23].

Bismuth (Bi) is the only element in the period 6 with an outer $6 s^{2}$ lone pair and low toxicity [24]. Bi is also the heaviest group $\mathrm{V}$ element and exhibits strong spin-orbit coupling (SOC), which often determines the relevant features of the band structure [25]. Such similarity with $\mathrm{Pb}$ makes $\mathrm{Bi}$ an attractive element to develop new compounds for PV applications, and in fact, Bi-based ternaries have been recently the subject of both theoretical and experimental work. These include halide-containing materials such as (MA) ${ }_{3} \mathrm{Bi}_{2} \mathrm{I}_{9}$, $\mathrm{Cs}_{3} \mathrm{Bi}_{2} \mathrm{I}_{9}$, BiSI and $\mathrm{BiOI}$, and some chalcogenides, $\mathrm{AgBiS}_{2}$ [26-29]. Some of the halide-containing compounds, namely (MA) $)_{3} \mathrm{Bi}_{2} \mathrm{I}_{9}, \mathrm{Cs}_{3} \mathrm{Bi}_{2} \mathrm{I}_{9}$ and $(\mathrm{MA})_{3} \mathrm{Bi}_{2} \mathrm{I}_{9} \mathrm{Cl}_{x}$, have been tested for light absorption [30-32] but unfortunately only display modest efficiencies ranging between $0.1 \%$ and $1 \%$.

An interesting chemical possibility that certainly may improve the materials' structural and the thermodynamic stability is that of replacing the halide anion with a chalcogen element such as $\mathrm{O}$ and $\mathrm{S}$. One has then the option to form ternaries with either $1: 1: 2$ or $1: 1: 3$ stoichiometric ratios, whose prototypes are $\mathrm{CsBiO}_{2}$ and $\mathrm{CsBiO}_{3}$, respectively, and where $\mathrm{Bi}$ is either in the +3 or +5 oxidation state. Several compounds in this class have been synthesized and experimentally characterized with piezoelectric and photocatalytic applications. For some of them, theoretical work also exists. These include $\mathrm{NaBiO}_{2}$ [33], $\mathrm{KBiO}_{2}$ [34], $\mathrm{RbBiO}_{2}$ [33,34], $\mathrm{CsBiO}_{2}$ [33], $\mathrm{NaBiO}_{3}$ [35-38], $\mathrm{KBiO}_{3}$ [39], $\mathrm{NaBiS}_{2}$ [40,41], and $\mathrm{KBiS}_{2}[40,41]$.

In the present work, we explore theoretically the potential of this class of ternary Bi-based chalcogenides for PV applications, by exploring their structural, thermodynamic, mechanical, elastic, electronic, and dielectric properties. For all compounds, the computed optimal crystal structure is found to be consistent with available experimental data. The only exception is for $\mathrm{NaBiS}_{2}$ and $\mathrm{KBiS}_{2}$, which are calculated trigonal instead of cubic as reported in the literature. This difference is attributed to the site occupation disorder in the experimental samples. The theoretical and experimental data can be reconciled by assuming that the experimental structure is the result of a macroscopic average of various equivalent trigonal orientations. All compounds are indirect gap semiconductors with the exception of $\mathrm{NaBiO}_{2}$ and $\mathrm{KBiO}_{2}$.

The paper is organized as follows. Section II discusses the computational methods used for the calculations and our strategy for constructing materials prototypes. In Secs. III A, III B, III C, III D, and III E, we present our results alongside a discussion of the structural, thermodynamic, mechanical, elastic, electronic, and dielectric properties of all compounds, respectively. Finally, in Sec. IV we summarize our finding and highlight possible future directions.

\section{METHODS}

\section{A. Computational details}

Structural optimization and the calculation of the electronic structure and dielectric properties have been performed by density functional theory (DFT) as implemented in the allelectron code FHI-AIMS (Fritz Haber Institute Ab Initio Molecular Simulations) [42]. The Perdew-Burke-Ernzerhof (PBE) [43] parametrization of the generalized gradient approximation (GGA) of the exchange-correlation energy has been used throughout. FHI-AIMS writes the electron density and all the operators over an all-electron numerical-atomicorbitals basis set, and here we have used the 'tight' option, which provides safe pre-constructed default definitions for the different chemical species (elements). The Broyden-FletcherGoldfarb-Shanno (BFGS) algorithm [44] has been used to relax both the atomic coordinates and the lattice vectors of all the ternary compounds, with convergence cutoffs of $10^{-3} \mathrm{eV}$, $10^{-4} \mathrm{eV}$, and $10^{-3} \mathrm{eV} / \AA$, respectively, for the Kohn-Sham eigenvalues, the total energy, and the atomic forces. The reciprocal space integration has been performed over a $8 \times 8 \times 8$ grid .

In general, the role of van der Waals interactions in solids is still a matter of discussion [45], although in some cases they provide an overall improvement of the cohesive properties of ionic and semiconductor solids [46], and there is evidence that these contribute in determining the structure of HOIPs [47]. The GGA-PBE functional does not describe weak dispersive forces. We remedy this shortfall by including the TkatchenkoScheffler van der Waals correction scheme [48], when performing the structural relaxation. Spin-orbit coupling (SOC) is considered in the calculation of the electronic structure.

The linear macroscopic dielectric tensor, $\varepsilon_{i j}(\omega)$, is calculated within the random phase approximation (RPA) framework as reported by Ambrosch-Draxl et al. [49]. The frequency-dependent imaginary part of the interband contribution to the linear dielectric tensor is computed with FHIAIMS as

$$
\begin{aligned}
\operatorname{Im}\left(\varepsilon_{i j}(\omega)\right)= & \frac{1}{\pi \omega^{2}} \sum_{n, n^{\prime}} \int_{\vec{k}} p_{i ; n^{\prime}, n, \vec{k}} p_{j ; n^{\prime}, n, \vec{k}}\left[f_{0}\left(\varepsilon_{n, \vec{k}}\right)\right. \\
& \left.-f_{0}\left(\varepsilon_{n^{\prime}, \vec{k}}\right)\right] \delta\left(\varepsilon_{n^{\prime}, \vec{k}}-\varepsilon_{n, \vec{k}}-\omega\right),
\end{aligned}
$$

where $\varepsilon_{n, \vec{k}}$ is the eigenvalue of the Kohn-Sham (KS) eigenstate of band index $n$ and wave vector $\vec{k}, f_{0}\left(\varepsilon_{n, \vec{k}}\right)$ is the Fermi function with $\varepsilon_{F}$ being the chemical potential, $\delta\left(\varepsilon_{n^{\prime}, \vec{k}}-\varepsilon_{n, \vec{k}}-\right.$ $\omega)$ is the Dirac function (approximated as a Gaussian), and $p_{j ; n^{\prime}, n, \vec{k}}$ are the momentum matrix elements calculated from the real-space basis functions in $k$ space and the KS-eigenstate basis. The broadening values for the Fermi and Gaussian functions are set as $0.001 \mathrm{eV}$ and $0.02 \mathrm{eV}$, respectively. The dielectric tensor thus obtained is hermitian and the number of linearly independent components depends on the symmetry of the crystal. By using the Kramers-Kronig transformation, the corresponding real parts can be obtained. Since the calculation of the density of states (DOS) and the dielectric function are very sensitive to the $k$-point grid, grids as large as $15 \times 15 \times$ 15 and $10 \times 10 \times 10$ are used for $A \mathrm{Bi} X_{2}$ and $A \mathrm{Bi} X_{3}$ type compounds, respectively. 
Throughout this project we have also used the Vienna $a b$ initio simulation package (VASP) [50]. VASP implements DFT within the projector-augmented wave method [51] and it has been utilized for a few test calculations and to compute the elastic constants of the materials under consideration. All VASP calculations are at the PBE-GGA level and the FHIAIMS optimized structures have been used to compute the elastic constants.

In order to correct the systematic band gap underestimation of the GGA, we have performed calculations using the Gritsenko, van Leeuwen, van Lenthe, and Baerends functional (GLLB) [52], as implemented in the GPAW code [53]. Its optimization for solids (GLLB-SC) [54] has been reported to predict band gaps in good agreement with experiments [55]. The GLLB-SC functional presents a computational cost significantly lower than that of hybrid functionals without compromising on the accuracy [56]. Hence, GLLB-SC has been chosen to correct the band gaps of the $\mathrm{Bi}$ chalcogenides investigated here.

Finally, the dynamic stability of some of the prototypes required to be validated. For this purpose, we have carried out phonon band structure calculations using the finite displacement method as implemented in the Phonopy package [57]. In particular, we have used a $2 \times 2 \times 2$ supercell and a $6 \times 6 \times 6$ $k$-grid mesh.

\section{B. Construction of the prototypes}

From our literature survey of Bi-containing ternary compounds, we have decided to focus on the two stoichiometry compositions $A \mathrm{Bi} X_{2}$ and $A \mathrm{Bi} X_{3}$, where $A=\mathrm{Na}, \mathrm{K}$ and $X=$ $\mathrm{O}, \mathrm{S}$. These compositions appear promising to host suitable semiconductors for PV applications, with the finite band gap and robust structural stability. In the case of the oxides, crystallographic data exist for $\mathrm{NaBiO}_{2}, \mathrm{NaBiO}_{3}$, and $\mathrm{KBiO}_{2}$. Therefore, their initial structure has been taken from the Materials Project Library [58]. The $2 \times 1 \times 1 \mathrm{NaBiO}_{2}$ supercell from the Materials Project Library has been chosen to match the lattice parameters of the experimentally reported structure [33]. In contrast, $\mathrm{KBiO}_{3}$ has been claimed to crystallize in the cubic crystal system with space group $\operatorname{Im} \overline{3}$ (No. 204), although no crystallographic data are available. The closest prototype included in the crystallographic database is the one lying in the space group, $\operatorname{Pn} \overline{3}$ (No. 201), whose primitive cell contains 60 atoms. Alternatively, one can use the experimental structure of $\mathrm{NaBiO}_{3}$ as a prototype for $\mathrm{KBiO}_{3}$ (30 atoms per cell). By comparing the total energy of the final structures relaxed from these two prototypes, we find the second one to be most stable. Therefore, this is the structure that we investigate further. All these structures have been relaxed with GGA-PBE, including van der Waals corrections. Then, we have replaced in the final structures the $\mathrm{O}$ ions with $\mathrm{S}$, so as to generate the prototypes for $\mathrm{NaBiS}_{3}$ and $\mathrm{KBiS}_{3}$. These compounds also have been fully relaxed.

If one employs the same strategy for $\mathrm{NaBiS}_{2}$ and $\mathrm{KBiS}_{2}$, the resulting prototypes will belong to the tetragonal and monoclinic system, respectively. However, it has been reported that there exist for both high-symmetric cubic structures [59], which unfortunately are not available in any of the crystal structure databases. As such, together with the tetragonal and monoclinic structures, we have also constructed cubic prototypes of $\mathrm{NaBiS}_{2}$ and $\mathrm{KBiS}_{2}$. These are obtained by modifying the rocksalt structure according to the prescription adopted in Ref. [60] for ternary $A P n E_{2}$ compounds $(A=\mathrm{Na}$, $\mathrm{K}, \mathrm{Ag} ; P n=\mathrm{As}, \mathrm{Sb}, \mathrm{Bi} ; E=\mathrm{S}, \mathrm{Se}, \mathrm{Te})$.

The thermodynamic stability of all compounds investigated has been assessed by computing the convex hull diagrams and the possible decomposition reaction pathways. In this case, we have considered decompositions along with binary phase with the crystal structure of such binaries extracted from the AFLOWLIB.org library [61,62]. Finally, the $k$-point paths in reciprocal space used to compute the band structure have been obtained by using the aflow-online tool [63].

\section{RESULTS AND DISCUSSION}

We present here the structural, thermodynamic, elastic, mechanical, electronic, and dielectric properties of the two classes of alkali-metal Bi-based ternary chalcogenides, $A \mathrm{Bi} X_{2}$ and $\mathrm{ABi}_{3}$. Here, $A$ is a monovalent $\mathrm{Na}$ or $\mathrm{K}$ cation and $X$ is the divalent $\mathrm{O}$ or $\mathrm{S}$ anion, thus that $\mathrm{Bi}$ is either in the +3 or +5 oxidation state.

\section{A. Structural properties}

The computed cell parameters, lattice system, and space group of the ternary $\mathrm{Bi}$-chalcogenides after relaxation are tabulated in Table I, while their corresponding ball-and-stick structural diagrams are presented in Fig. 1. Let us discuss the oxides first. $\mathrm{NaBiO}_{2}$ is calculated to be a monoclinic crystal with the $C 2 / c$ space group, consistent with available experiments [33]. The computed lattice constants are in close agreement with crystallographic data, except for the $c$ parameter, which is overestimated (5.97 $\AA$ against $5.88 \AA$ ) as sometimes expected from the GGA. In $\mathrm{NaBiO}_{2}$, the $\mathrm{Bi}$ cations are bonded to four $\mathrm{O}$ atoms in a tetrahedral local coordination (see polygons diagrams in Fig. 1). When the lone pair is taken into consideration, $\mathrm{Bi}$ could be assigned to a trigonal bipyramidal molecular geometry. In such $C 2 / c$ structure, $\mathrm{Na}$ is found in octahedral coordination. In contrast, $\mathrm{NaBiO}_{3}$, where the $\mathrm{Bi}$ oxidation state becomes +5 , has a trigonal layered structure and it is found in the much more symmetric $R \overline{3}$ space group. Also in this case, there is good agreement between the computed structural parameters and those available from diffraction experiments [35], with an elongation of the planar lattice constant $a=b(5.69 \AA$ against the experimental value of $5.57 \AA$ ) and a compression of the out-of-plane one, $c(15.44 \AA$ against $15.99 \AA)$. In this case, both the $\mathrm{Na}$ and $\mathrm{Bi}$ cations have octahedral coordination with $\mathrm{O}$. The Na octahedra bond to each other via edge sharing, and so do the Bi ones. Then, there exist both corner-sharing and face-sharing coordination between the $\mathrm{Na}$ and $\mathrm{Bi}$ octahedra. In general, this structure appears to be made of $\mathrm{BiO}_{6}$ octahedra monolayers separated by intercalated $\mathrm{Na}$.

Moving to K-containing oxides, we find that $\mathrm{KBiO}_{2}$ crystallizes in a layered structure of the monoclinic system with $C 2 / c$ space group. This is also consistent with crystallographic data [64] although we find a rather large deviation between one of the calculated lattice parameters, $c=6.55 \AA$, with respect to its corresponding experimental value (5.97 $\AA$ ). 
TABLE I. Structural parameters of the eight compounds investigated after full structural relaxation. In the table, we report the number of atoms per unit cell $N_{\mathrm{a}}$, the calculated lattice parameters $(a, b, c)$, the lattice vector angles $(\alpha, \beta, \gamma)$, the crystal structure system (CSS), the space group (SG) with the space group number in bracket, the atomization energy $\Delta E_{\mathrm{a}}$, and the decomposition energy $\Delta E_{\mathrm{d}}$.

\begin{tabular}{|c|c|c|c|c|c|c|c|c|c|c|c|}
\hline \multirow[b]{2}{*}{ Compound } & \multirow[b]{2}{*}{$N_{\mathrm{a}}$} & \multicolumn{3}{|c|}{$(a, b, c)(\AA)$} & \multicolumn{3}{|c|}{$(\alpha, \beta, \gamma)\left(^{\circ}\right)$} & \multirow[b]{2}{*}{ CSS } & \multirow[b]{2}{*}{ SG } & \multirow[b]{2}{*}{$\Delta E_{\mathrm{a}}(\mathrm{eV})$} & \multirow[b]{2}{*}{$\Delta E_{\mathrm{d}}(\mathrm{eV})$} \\
\hline & & $a$ & $b$ & $c$ & $\alpha$ & $\beta$ & $\gamma$ & & & & \\
\hline $\mathrm{NaBiO}_{2}$ & 16 & 7.38 & 7.32 & 5.97 & 90.0 & 127.0 & 90.0 & Monoclinic & $C 2 / c(15)$ & -4.18 & 0.29 \\
\hline $\mathrm{NaBiO}_{3}$ & 30 & 5.69 & 5.69 & 15.44 & 90.0 & 89.9 & 119.9 & Trigonal & $R \overline{3}(148)$ & -4.29 & 0.51 \\
\hline $\mathrm{KBiO}_{2}$ & 16 & 7.84 & 7.89 & 6.55 & 89.9 & 131.5 & 90.0 & Monoclinic & $C 2 / c(15)$ & -4.60 & 0.33 \\
\hline $\mathrm{KBiO}_{3}$ & 30 & 5.80 & 5.80 & 17.55 & 90.0 & 89.9 & 119.9 & Trigonal & $R \overline{3}$ & -4.43 & 0.36 \\
\hline $\mathrm{NaBiS}_{2}$ & 16 & 7.90 & 8.08 & 7.90 & 60.8 & 60.0 & 60.8 & Trigonal & $R \overline{3} m$ & -4.54 & 0.47 \\
\hline $\mathrm{NaBiS}_{3}$ & 30 & 6.39 & 7.66 & 18.45 & 81.1 & 86.0 & 121.3 & Triclinic & $P \overline{1}(2)$ & -3.50 & 0.47 \\
\hline $\mathrm{KBiS}_{2}$ & 16 & 7.93 & 8.65 & 7.93 & 62.7 & 60.0 & 62.7 & Trigonal & $R \overline{3} m$ & -4.78 & 0.45 \\
\hline $\mathrm{KBiS}_{3}$ & 30 & 6.34 & 8.08 & 17.43 & 91.8 & 73.5 & 114.5 & Triclinic & $P \overline{1}(2)$ & -3.61 & 0.42 \\
\hline
\end{tabular}

Such deviation translates into a deviation of the nonright angle, which is calculated at $131.5^{\circ}$ against the experimental assignment at $124.9^{\circ}$. We have repeated the calculation with VASP and obtained similar results for the GGA, which is then the most probable origin of the disagreement with ex- periments. The presence of the Bi lone pair results in a seesaw molecular geometry, where the $\mathrm{Bi}$ local coordination has the $C_{2 v}$ point group. The same local geometry is found also for $\mathrm{K}$. The so-formed $\mathrm{Bi}$ and $\mathrm{K}$ trigonal bipyramids are found to share edges. Finally, for $\mathrm{KBiO}_{3}$ geometry relaxation from the

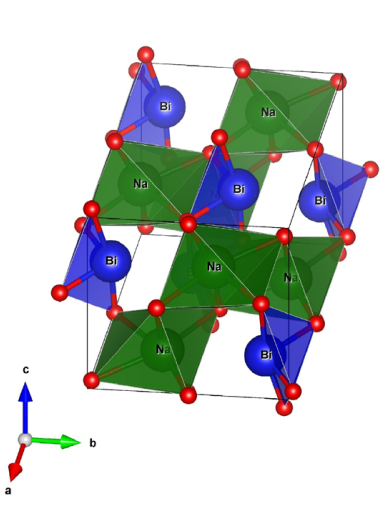

(a) $\mathrm{NaBiO}_{2}$

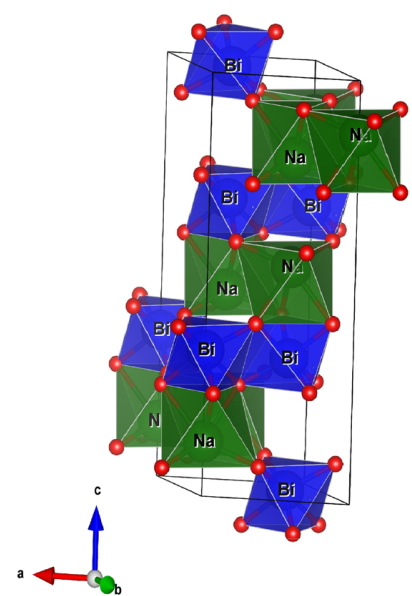

(e) $\mathrm{NaBiO}_{3}$

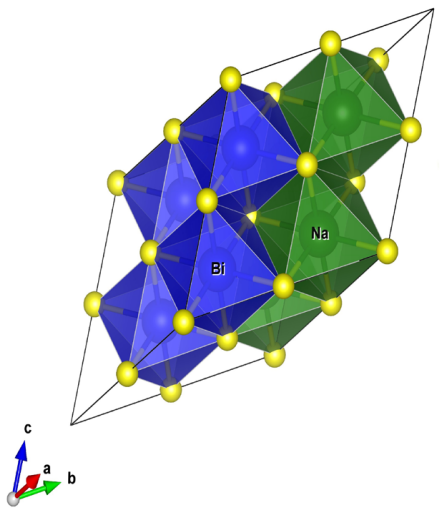

(b) $\mathrm{NaBiS}_{2}$

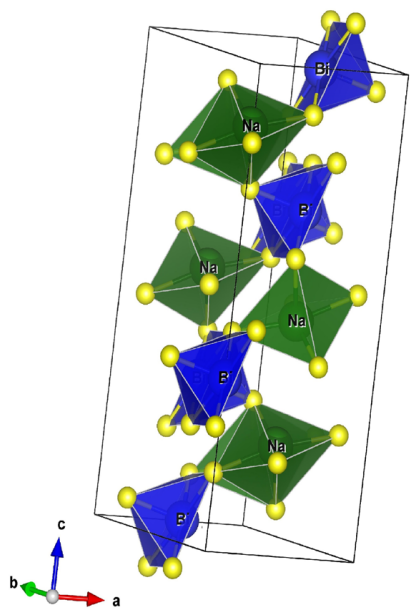

(f) $\mathrm{NaBiS}_{3}$

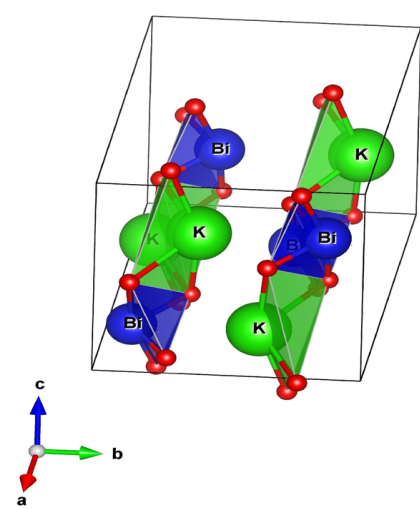

(c) $\mathrm{KBiO}_{2}$

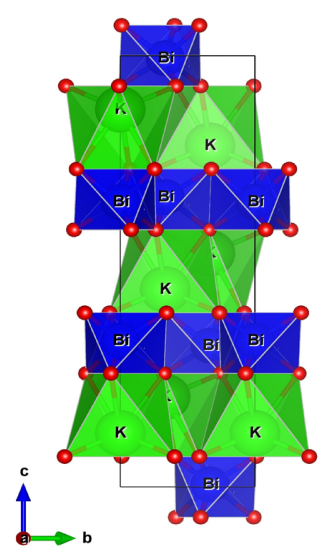

(g) $\mathrm{KBiO}_{3}$

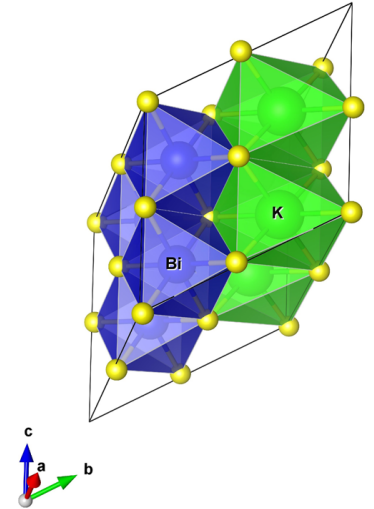

(d) $\mathrm{KBiS}_{2}$

FIG. 1. The crystal structure of (a) $\mathrm{NaBiO}_{2}$, (b) $\mathrm{NaBiS}_{2}$, (c) $\mathrm{KBiO}_{2}$, (d) $\mathrm{KBiS}_{2}$, (e) $\mathrm{NaBiO}_{3}$, (f) $\mathrm{NaBiS}_{3}$, (g) $\mathrm{KBiO}_{3}$, and (h) $\mathrm{KBiS}_{3}$. The experimental unit cells of (a) $\mathrm{NaBiO}_{2}$, (c) $\mathrm{KBiO}_{2}$, (e) $\mathrm{NaBiO}_{3}$, and (g) $\mathrm{KBiO}_{3}$ have been taken from the Materials Project database [58] (see details concerning $\mathrm{KBiO}_{3}$ in the main text). The initial structures of (b) $\mathrm{NaBiS}_{2}$, (d) $\mathrm{KBiS}_{2}$, (f) $\mathrm{NaBiS}_{3}$, and (h) $\mathrm{KBiS}_{3}$ have been obtained by replacing $\mathrm{O}$ with $\mathrm{S}$ in the corresponding oxides (see main text for more details on how the prototypes of $\mathrm{NaBiS}_{2}$ and $\mathrm{KBiS}_{2}$ have been constructed). Color code: $\mathrm{Bi}=$ blue, $\mathrm{O}=$ red, $\mathrm{S}=$ yellow, $\mathrm{Na}=$ green, $\mathrm{K}=$ light green. The colored polygons indicate the local coordination of $\mathrm{Bi}$ (green) and the alkali metal cation (blue). 
parental $\mathrm{NaBiO}_{3}$ structure leaves the crystal in the original trigonal $R \overline{3}$ space group, and the only notable difference between $\mathrm{NaBiO}_{3}$ and $\mathrm{KBiO}_{3}$ is that the latter presents a significantly larger $c / a$ ratio (3.03 for $\mathrm{KBiO}_{3}$ against 2.71 for $\mathrm{NaBiO}_{3}$ ). Again, the overall structure can be viewed as made of planes of $\mathrm{BiO}_{6}$ octahedra separated by intercalated $\mathrm{K}$. This presents analogies with the $\beta-\mathrm{Sb}_{2} \mathrm{O}_{4}$ structure found for the mixed-valence $\mathrm{Bi}_{2} \mathrm{O}_{4}$ compound [65].

Given the large electronegativity of $\mathrm{O}$ with respect to that of $\mathrm{Bi}$, it is expected that $\mathrm{Bi}$-containing oxides may present large band gaps. A possible strategy to reduce such a band gap is that of replacing $\mathrm{O}$ with the less electronegative $\mathrm{S}$, a solution that may also result in more structurally stable compounds $[66,67]$. In general, there is evidence of band-gap reduction achieved by $\mathrm{O}$ to $\mathrm{S}$ substitution in perovskites $[68,69]$, and this is attributed to two effects. The presence of the empty $\mathrm{S} 3 d$ orbitals contributes to lower down the energy of the conduction band, an effect not present for O. On the other hand, the larger ionic radius of $\mathrm{S}^{2-}(0.184 \mathrm{~nm})$ compared to $\mathrm{O}^{2-}$ $(0.140 \mathrm{~nm})$ results in a larger cell volume and consequently in less dispersive bands.

As mentioned before, for $\mathrm{NaBiS}_{2}$ we have constructed the prototype either by replacing $\mathrm{O}$ with $\mathrm{S}$ in the previously relaxed $\mathrm{NaBiO}_{2}$ structure or by randomly replacing the $\mathrm{Na}$ sites of a $\mathrm{NaCl}$ rocksalt supercell with a random distribution of $\mathrm{Na}$ and $\mathrm{Bi}$ (the $\mathrm{Cl}$ sites are all replaced by $\mathrm{S}$ ) [60]. In this second case, we have employed $2 \times 2 \times 2$ supercells of the rocksalt primitive cell and explored different $\mathrm{Na} / \mathrm{Bi}$ distributions. Geometrical relaxation starting from the first prototype yields a body-centered tetragonal crystal structure $\left(I 4_{1} /\right.$ amd $)$, while that originating from the second converges to a trigonal crystal system, with the structure displayed in Fig. 1(b). This latter is lower in energy and therefore it is selected as our final ground-state structure. In such structure, the $\mathrm{Na}$ - and $\mathrm{Bi}$-centered octahedra are connected by sharing edges.

Note that the fine details of the structures obtained by relaxing the Na-type $2 \times 2 \times 2$ supercell depend on the specific arrangement of the $\mathrm{Na}$ and $\mathrm{Bi}$ ions. Namely, although the structural relaxation begins from identical supercells with lattice parameter $a=7.98 \AA$ and angle $\alpha=60^{\circ}$, the final geometry depends on the specific arrangement of $\mathrm{Na}$ and $\mathrm{Bi}$. In particular, the energy minimum is threefold degenerate depending on the elongated lattice vector. If one assumes that the actual structure is the result of an average of such degenerate ground states (note that other nondegenerate configurations are also rather close in energy) and that the average lattice vector is simply the algebraic average of the computed lattice vectors for the $R \overline{3} m$ structure, then we obtain an equivalent rocksalt lattice parameter of $5.63 \AA$. This is consistent with an old experimental determination of $5.77 \AA$ [59]. We then conclude that actual $\mathrm{NaBiS}_{2}$ samples are possibly disordered and that the disorder- and thermal-averaged structure is actually cubic, although locally the $R \overline{3} \mathrm{~m}$ space group is adopted. This is a situation similar to that encountered in halide perovskites, where the high symmetry structure is the result of a macroscopic average of lower-symmetry ones [70].

In contrast, there is no experimental determination for $\mathrm{NaBiS}_{3}$, which is predicted triclinic with space group $P \overline{1}$ [see Fig. 1(f)]. In $\mathrm{NaBiS}_{3}$, the $\mathrm{Bi}$ cations are bonded to five $\mathrm{S}$ anions in a square pyramidal molecular geometry and the $\mathrm{Na}$ ones are bonded to five $\mathrm{S}$ anions in an irregular tetrahedral molecular geometry.

Moving to Bi compounds containing both $\mathrm{S}$ and $\mathrm{K}$, we have treated $\mathrm{KBiS}_{2}$ in a similar way as $\mathrm{NaBiS}_{2}$. Also, in this case relaxation starting from the parental $\mathrm{KBiO}_{2}$ structure converges to a monoclinic crystal with $C 2 / c$ space group, namely, it remains with the same structure of $\mathrm{KBiO}_{2}$. However, relaxation starting from the substituted rocksalt $2 \times 2 \times 2$ supercell returns us a lower energy trigonal geometry with space group $R \overline{3} m$ and an internal arrangement of $\mathrm{K}$ and $\mathrm{Bi}$ identical to that of $\mathrm{Na}$ and $\mathrm{Bi}$ for $\mathrm{NaBiS}_{2}$ [see Fig. 1(d)]. Again $\mathrm{K}$ and $\mathrm{Bi}$ are sixfold bonded to $\mathrm{S}$ in an octahedral molecular geometry and the octahedra are edge sharing. The same considerations made for $\mathrm{NaBiS}_{2}$ remain valid here and the average cubic structure is found at a lattice constant of $5.78 \AA$, to be compared with the experimental estimate of $6.04 \AA$ [59].

The dynamical stability of the cubic and trigonal phases of $\mathrm{NaBiS}_{2}$ and $\mathrm{KBiS}_{2}$ have been further investigated by calculating their phonon spectra (see Figs. S1 and S2 of the SM [71]). For $\mathrm{KBiS}_{2}$, we have found the cubic phase to be unstable, with imaginary phonon modes across the entire Brillouin zone, while the trigonal phase displays only a tiny region of imaginary frequencies near to the $\Gamma$ point, which is attributed to a small numerical error. In contrast, both the cubic and trigonal phases of $\mathrm{NaBiS}_{2}$ appear stable (both phonon spectra show a small instability at $\Gamma$ due to numerical errors). As such, we conclude that the experimentally measured cubic phases of $\mathrm{NaBiS}_{2}$ and $\mathrm{KBiS}_{2}$ are the result of a macroscopic average. In the case of $\mathrm{KBiS}_{2}$, this includes differently oriented trigonal phases, while in that of $\mathrm{NaBiS}_{2}$ it is possible that some local cubic arrangement is also found.

Finally, the crystal structure of $\mathrm{KBiS}_{3}$ is found to be triclinic with $P \overline{1}$ space group [Fig. 1(h)], namely it has a lower symmetry than its parental trigonal, $\mathrm{KBiO}_{3}$. This is the material presenting the lowest degree of bonding. The $\mathrm{K}$ and $\mathrm{Bi}$ cations are coordinated to four $\mathrm{S}$ anions in a seesaw molecular geometry.

\section{B. Thermodynamic stability}

Several quantities may provide information about the thermodynamic stability of the compounds investigated. The atomization energy (per atom) is defined as

$$
\Delta E_{\mathrm{a}}=E_{\mathrm{total}} / N-\sum_{i} x_{i} E_{i},
$$

where $E_{\text {total }}$ is the total energy per formula unit of the ternary compound, $E_{i}$ is the atomic energy of the element $i, x_{i}$ is the atomic fraction, and $N$ is the total number of atom per formula unit. This gives us information about the total bond energy of a solid but says little about its stability. A better quantity is provided by the decomposition energy $\Delta E_{\mathrm{d}}$. It is defined as the total energy difference, per atom in the formula unit, of a given ternary against the binary products of a balanced decomposition reaction, minimized over all possible binaries choices. The decomposition energy thus writes

$$
\Delta E_{\mathrm{d}}=\operatorname{Min}\left[\sum_{i} c_{i} E_{i}^{\mathrm{binary}} / N_{i}\right]-E^{\text {ternary }} / N,
$$


where $E^{\text {ternary }}$ is the energy per formula unit of the ternary, $E_{i}^{\text {binary }}$ is the energy per formula unit of the binary, $c_{i}$ are the fractional coefficients needed to balance the reaction, and $N$ and $N_{i}$ are the total number of atom per formula unit of the ternary and the $i$ th binary, respectively.

With this definition, a ternary compound is stable when $\Delta E_{\mathrm{d}}>0$, namely when there is no energetically favorable decomposition reaction. In contrast, when $\Delta E_{\mathrm{d}}<0$ there exists at least one decomposition path whose energy is lower than that of the ternary material under investigation, namely the ternary is thermodynamically unstable against that particular reaction product. Note that in this case, the ternary can still be stabilized by the reaction kinetics, meaning that it can still be metastable. Note also that our definition of $\Delta E_{\mathrm{d}}$ does not consider decomposition channels involving alternative ternaries together with the binaries, but it provides a much more stringent criterion for stability than the atomization energy alone [72]. It is, however, unlikely that for any given ternary compound a ternary decomposition path can present a lower total energy than those involving competing binaries, since the enthalpy gain in general decreases with the number of elements contained in a compound [73]. In computing $\Delta E_{\mathrm{d}}$, we have taken the binary prototypes from the Materials Project library [58]. These have been fully relaxed and their electronic structure computed. Note that in the case of polymorphism we have always considered the binary with the lowest energy. Finally, $\Delta E_{\mathrm{d}}$ has been calculated using the Pymatgen library [74-76].

Our results are reported in Table I, while the list of the most stable decomposition of each of the eight compounds is presented below.

$$
\begin{aligned}
& \mathrm{NaBiO}_{2} \rightarrow \frac{1}{2} \mathrm{Na}_{2} \mathrm{O}+\frac{1}{2} \mathrm{Bi}_{2} \mathrm{O}_{3}, \\
& \mathrm{NaBiO}_{3} \rightarrow \mathrm{NaO}+\mathrm{BiO}_{2}, \\
& \mathrm{KBiO}_{2} \rightarrow \frac{1}{2} \mathrm{~K}_{2} \mathrm{O}+\frac{1}{2} \mathrm{Bi}_{2} \mathrm{O}_{3}, \\
& \mathrm{KBiO}_{3} \rightarrow \frac{1}{4} \mathrm{KO}_{2}+\frac{3}{4} \mathrm{KO}+\frac{1}{4} \mathrm{Bi}_{4} \mathrm{O}_{7} \text {, } \\
& \mathrm{NaBiS}_{2} \rightarrow \frac{1}{2} \mathrm{Na}_{2} \mathrm{~S}+\frac{1}{2} \mathrm{Bi}_{2} \mathrm{~S}_{3}, \\
& \mathrm{NaBiS}_{3} \rightarrow \frac{1}{2} \mathrm{NaS}_{2}+\frac{1}{2} \mathrm{NaS}+\frac{1}{2} \mathrm{Bi}_{2} \mathrm{~S}_{3}, \\
& \mathrm{KBiS}_{2} \rightarrow \frac{1}{2} \mathrm{~K}_{2} \mathrm{~S}+\frac{1}{2} \mathrm{Bi}_{2} \mathrm{~S}_{3}, \\
& \mathrm{KBiS}_{3} \rightarrow \frac{1}{2} \mathrm{~K}_{2} \mathrm{~S}_{3}+\frac{1}{2} \mathrm{Bi}_{2} \mathrm{~S}_{3} .
\end{aligned}
$$

In general, we find that for all compounds $\Delta E_{\mathrm{d}}$ is positive, meaning that they are all stable, as expected from experimental evidence. In the case of oxides, the $A \mathrm{BiX}_{3}$ stoichiometry is more robust against decomposition than the $A \mathrm{BiX}_{2}$ one, suggesting that in this class of materials $\mathrm{Bi}$ has a preference for the +5 oxidation state, despite that most of the Bi chemistry [77] is based on the +3 . Such difference is significantly more pronounced for $\mathrm{Na}$-containing compounds than K-containing ones. The same is not valid for sulphides, for which the two stoichiometries have essentially identical stability.

\section{Elastic and mechanical properties}

The elastic stiffness constants $C_{i j}$ are calculated for all the compounds in order to determine the bulk modulus $B$, the shear modulus $G$, the Young's modulus $Y$, and the Poisson's ratio $v$. Unfortunately, to the best of our knowledge, experimental and theoretical elastic constants for the eight alkali-metal based bismuth chalcogenides are not available, so that our calculations cannot be compared with literature and constitute the first assessment made of the elastic properties of this class of materials. We have then used the $C_{i j}$ 's to evaluate the mechanical stability of our compounds according to the Born elastic stability criteria [78,79].

Let us start our discussion from the mechanical stability analysis. The eight compounds investigated fall into three crystal structure systems, namely the trigonal, the monoclinic, and the triclinic, with the Born elastic stability conditions varying for the different crystal lattices. The monoclinic and triclinic structures have 13 and 21 independent elastic constants, respectively. The stability conditions for these two crystal systems could be evaluated by demonstrating that the leading principal minors of the elastic stiffness constants matrix are positive [80]. Such condition for the trigonal structure at zero pressure, for instance, reads

$$
\begin{gathered}
C_{11}>\left|C_{12}\right|, C_{11}, C_{33}, C_{44}>0, \\
{\left[\left(C_{11}+C_{12}\right) C_{33}-2 C_{13}^{2}\right]>0,} \\
{\left[\left(C_{11}-C_{12}\right) C_{44}-2 C_{14}^{2}\right]>0 .}
\end{gathered}
$$

The elastic stiffness constants of all the compounds are reported in Table S1 [71] of the Supplemental Material (SM). When these are input in the Born elastic stability criteria, we found that all compounds are mechanically stable at zero pressure.

Then $B, G, Y$, and $v$ can be computed directly from the elastic tensor. The explicit expressions for the trigonal system [81] are provided here in Eq. (5) through Eq. (10), while the corresponding ones for the monoclinic and triclinic systems can be easily found in literature [82,83]. In the definitions provided, the subscripts ' $\mathrm{V}$,' 'R,' and ' $\mathrm{H}$ ' indicate the Voigt, Reuss, and Hill approximations. The Voigt and Reuss equations relate $B$ and $G$ to the elastic constants $C_{i j}$ and their compliances $S_{i j}=C_{i j}^{-1}$, while the Hill approximation is the arithmetic mean of the Voigt and Reuss bounds [84,85]. It must be noted that the Voigt approximation provides an upper bound to the actual elastic moduli, while the Reuss one returns the lower limit.

$$
\begin{gathered}
B_{\mathrm{V}}=\frac{1}{9}\left(2 C_{11}+C_{33}\right)+\frac{2}{9}\left(C_{12}+2 C_{13}\right), \\
G_{\mathrm{V}}=\frac{1}{15}\left(2 C_{11}+C_{33}-C_{12}-2 C_{13}\right)+\frac{1}{5}\left(2 C_{44}+\frac{C_{11}-C_{12}}{2}\right) \\
G_{\mathrm{R}}=\frac{1}{4\left(2 S_{11}+S_{33}-S_{12}-2 S_{13}\right)+6\left(S_{44}+S_{11}-S_{12}\right)} \\
B_{\mathrm{R}}=\frac{15}{\left(2 S_{11}+S_{33}\right)+2\left(S_{12}+2 S_{13}\right)}, \\
Y=\frac{9 B_{\mathrm{H}} G_{\mathrm{H}}}{3 B_{\mathrm{H}}+G_{\mathrm{H}}}, \quad v=\frac{\left(3 B_{\mathrm{H}}-2 G_{\mathrm{H}}\right)}{2\left(3 B_{\mathrm{H}}+G_{\mathrm{H}}\right)}, \\
B_{\mathrm{H}}=\frac{1}{2}\left(B_{\mathrm{V}}+B_{\mathrm{R}}\right), \quad G_{\mathrm{H}}=\frac{1}{2}\left(G_{\mathrm{V}}+G_{\mathrm{R}}\right) .
\end{gathered}
$$


TABLE II. The bulk modulus $B$, shear modulus $G$, Young's modulus $Y$, Poisson's ratio $v$, and Pugh's ratio $\left(B_{\mathrm{H}} / G_{\mathrm{H}}\right)$ calculated for all the alkali-metal based bismuth ternary chalcogenides. The subscripts V, R, and H indicate the Voigt, Reuss, and Hill approximations, respectively.

\begin{tabular}{|c|c|c|c|c|c|c|c|c|c|}
\hline \multirow[b]{2}{*}{ Compound } & \multicolumn{3}{|c|}{ Bulk modulus (GPa) } & \multicolumn{3}{|c|}{ Shear modulus (GPa) } & \multirow{2}{*}{$\begin{array}{c}\text { Young's modulus (GPa) } \\
Y Y\end{array}$} & \multirow{2}{*}{$\begin{array}{c}\text { Poisson's ratio } \\
v\end{array}$} & \multirow{2}{*}{$\begin{array}{c}\text { Pugh's ratio } \\
\qquad B_{\mathrm{H}} / G_{\mathrm{H}}\end{array}$} \\
\hline & $B_{\mathrm{V}}$ & $B_{\mathrm{R}}$ & $B_{\mathrm{H}}$ & $G_{\mathrm{V}}$ & $G_{\mathrm{R}}$ & $G_{\mathrm{H}}$ & & & \\
\hline $\mathrm{NaBiO}_{2}$ & 45.57 & 27.47 & 36.52 & 24.42 & 1.07 & 12.74 & 34.25 & 0.34 & 2.87 \\
\hline $\mathrm{KBiO}_{2}$ & 41.46 & 32.13 & 36.80 & 21.51 & 1.17 & 11.34 & 30.85 & 0.36 & 3.25 \\
\hline $\mathrm{NaBiO}_{3}$ & 76.03 & 57.77 & 66.90 & 41.71 & 38.52 & 40.12 & 100.30 & 0.25 & 1.67 \\
\hline $\mathrm{KBiO}_{3}$ & 66.75 & 46.00 & 56.38 & 30.85 & 26.06 & 28.46 & 73.08 & 0.28 & 1.98 \\
\hline $\mathrm{NaBiS}_{2}$ & 55.68 & 54.23 & 54.96 & 38.28 & 32.73 & 35.50 & 87.64 & 0.23 & 1.56 \\
\hline $\mathrm{KBiS}_{2}$ & 57.54 & 57.27 & 57.40 & 40.89 & 29.84 & 35.36 & 88.02 & 0.24 & 1.62 \\
\hline $\mathrm{NaBiS}_{3}$ & 28.36 & 18.40 & 23.38 & 11.12 & 7.27 & 9.20 & 24.39 & 0.33 & 2.54 \\
\hline $\mathrm{KBiS}_{3}$ & 28.08 & 20.97 & 24.52 & 12.94 & 9.47 & 11.20 & 29.17 & 0.30 & 2.19 \\
\hline
\end{tabular}

Table II summarizes all the results. The bulk and shear moduli describe the hardness of a material, while the Young's modulus measures its stiffness [86]. The bulk, shear, and Young's moduli of the compounds investigated are found to decrease as the crystal symmetry is reduced, namely all compounds in the trigonal phase possess bulk, shear, and Young's moduli larger than those of the monoclinic and triclinic phases, with $\mathrm{NaBiO}_{3}$ and $\mathrm{KBiO}_{3}$ displaying the highest values of $B_{\mathrm{H}}, G_{\mathrm{H}}$, and $Y$. Interestingly, we find oxides to be stiffer than sulphides for the $\mathrm{ABi}_{3}$ stoichiometry, while the situation is reversed for the $A \mathrm{Bi} X_{2}$ one. Interestingly, $\mathrm{NaBiO}_{2}$ and $\mathrm{KBiO}_{2}$ exhibit the largest difference in the Voigt and Reuss bounds, while $\mathrm{NaBiS}_{2}$ and $\mathrm{KBiS}_{2}$ the smallest.

The Poisson's ratio serves as a valuable indicator of the plasticity of a material. When the value exceeds 0.26 , the material is predicted to be ductile, otherwise it is brittle [87]. Hence, it is found that all compounds in the trigonal phase, except $\mathrm{KBiO}_{3}$, are brittle, while all compounds with monoclinic and triclinic phase are ductile. The Pugh's $B / G$ ratio is another reference to predict the brittleness or ductility of a material [88]. If a material possesses a $B / G$ value larger than 1.75 , it is predicted ductile. On this ground, the brittleness of the compounds under investigation is found to reduce as one moves from the trigonal to the monoclinic and finally to the triclinic phase. $\mathrm{KBiO}_{2}$ is expected to have the highest ductility, while $\mathrm{NaBiS}_{2}$ to be the most brittle.

\section{Electronic structure properties}

Let us now discuss the features of the GGA band structures of the eight compounds investigated by starting from the oxides. In this section we will also report results obtained with the GLLB-SC functional, which improves the description of the band over the GGA. Since Na- and K-containing oxides have identical lattice structures with relatively similar lattice parameters, their electronic structure is very similar. Hence, we present, in Figs. 2 and 3, the band structure, respectively, of $\mathrm{NaBiO}_{2}$ and $\mathrm{NaBiO}_{3}$, while those of the corresponding $\mathrm{KBiO}_{2}$ and $\mathrm{KBiO}_{3}$ are displayed in the SM (Figs. S3 and S4, respectively [71]).

$\mathrm{NaBiO}_{2}$ and $\mathrm{KBiO}_{2}$ are two direct-band-gap semiconductors with the band gap located at the center of the Brillouin zone, $\Gamma$. Both the conduction and valence bands are rather dispersive around their edges, with the valence band presenting a region of low dispersion between $1.5 \mathrm{eV}$ and $2 \mathrm{eV}(1 \mathrm{eV}$ and
$1.5 \mathrm{eV})$ for $\mathrm{NaBiO}_{2}\left(\mathrm{KBiO}_{2}\right)$ below the valence band maximum (VBM). The projected density of state (PDOS) returns us a valence band mostly associated with $\mathrm{O}-p$ states, but with contributions also from $\mathrm{Bi}-s$ and $\mathrm{Bi}-p$ orbitals, fully consistent with the $\mathrm{O}^{2-}$ and $\mathrm{Bi}^{3+}$ oxidation states and with a significant $\mathrm{O}-p / \mathrm{Bi}-p$ orbital hybridization. In particular, the Bi-derived fraction of the PDOS is more pronounced at the VBM and it is responsible for the relatively large band dispersion, while the flat valence manifold is associated to the dominant $\mathrm{O}-p$ character. In contrast, the conduction band is mostly associated to $\mathrm{Bi}-p$, again with an hybridization contribution from the O-p orbital. The inclusion of spin-orbit coupling (SOC) in the calculation has little effect on the band structure, except for a marginal reduction of the conduction band bandwidth. This moves slightly the conduction band maximum (CBM) to higher energies, thus increasing the band gap. The GGA band gap of $\mathrm{NaBiO}_{2}\left(\mathrm{KBiO}_{2}\right)$ is around $1.35 \mathrm{eV}(1.80 \mathrm{eV})$, whereas its GLLB-SC counterpart is around $1.93 \mathrm{eV}(2.47 \mathrm{eV})$ (all the calculated band gaps are reported in Table III).

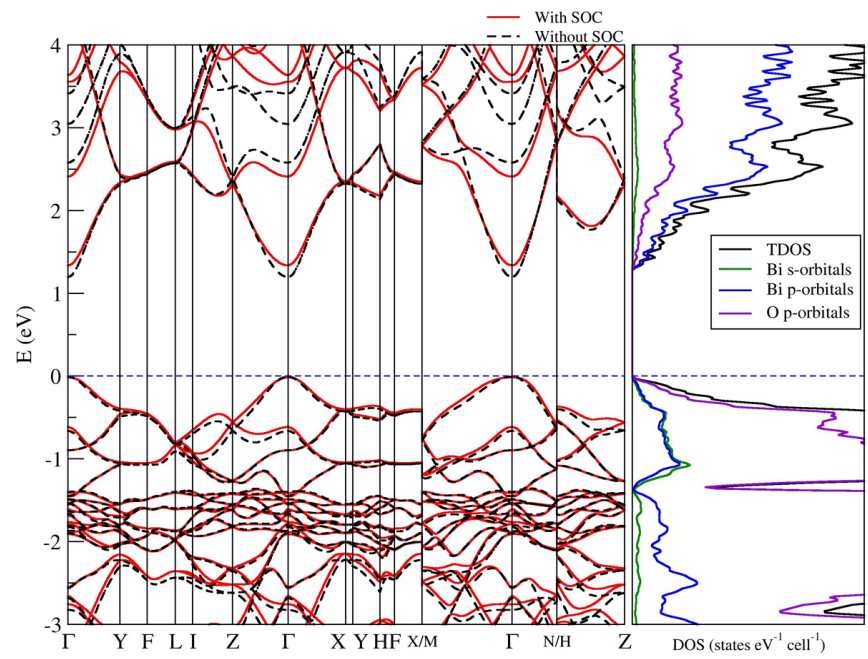

FIG. 2. The calculated DFT-GGA energy band structure and PDOS of $\mathrm{NaBiO}_{2}$. The VBM and CBM lie both at the $\Gamma$ point, making it a direct-gap semiconductor. The red and the dashed-black lines represent, respectively, the bands calculated with and without including spin-orbit coupling (SOC). The PDOS on the right-hand side is for the band structure calculated with SOC. 


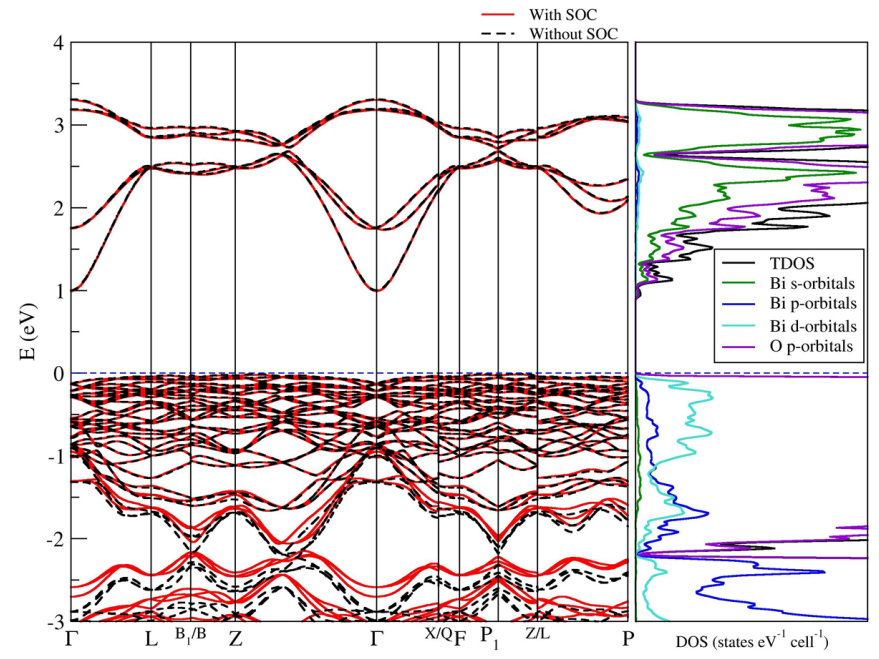

FIG. 3. The calculated DFT-GGA energy band structure and PDOS of $\mathrm{NaBiO}_{3}$. The compound is an indirect band-gap semiconductor with its VBM lying between $\mathrm{P}_{1}$ and $\mathrm{Z}$ and the CBM at $\Gamma$. The red and the dashed-black lines represent, respectively, the bands calculated with and without including spin-orbit coupling (SOC). The PDOS on the right-hand side is for the band structure calculated with SOC.

In contrast, both $\mathrm{NaBiO}_{3}$ (Fig. 3) and $\mathrm{KBiO}_{3}$ (Fig. S4 of the SM [71]) are calculated as indirect band-gap semiconductors with the CBM placed at $\Gamma$ and the VMB at a low-symmetry $k$ point located between $\mathrm{P}_{1}$ and $\mathrm{Z}$. The main feature of the band structure, however, is that the top of the valence band has very little dispersion, so that the direct and indirect band gaps are essentially identical. The rather flat VBM is completely determined by the $\mathrm{O}-p$ orbitals, while the hybridization contribution from the $\mathrm{Bi} s$ and $p$ shells is significantly reduced with respect to the case of the $A \mathrm{BiO}_{2}$ stoichiometry and it is evident only in the lower part of the valence manifold. This reflects the fact that now $\mathrm{Bi}$ is close to the nominal oxidation state of +5 (the $s$ shell is empty). A second important feature is that also the conduction band has an O- $p$ character, indicating that not all the $\mathrm{O}$ ions are saturated. The $\mathrm{Bi}-p$ empty conduction band (not displayed here) is well separated from the O- $p$ one and is placed at energies between $6 \mathrm{eV}$ and $8 \mathrm{eV}$ above the Fermi level (for both $\mathrm{NaBiO}_{3}$ and $\mathrm{KBiO}_{3}$ ).

As a consequence, the band gaps of the $A_{\mathrm{BiO}}$ compounds are between O- $p$ states and are lower than their $A \mathrm{BiO}_{2}$ counterparts. We obtain the PBE (GLLB-SC) gaps of $1.02 \mathrm{eV}$ $(1.80 \mathrm{eV})$ and $1.17 \mathrm{eV}(1.82 \mathrm{eV})$, respectively, for $\mathrm{NaBiO}_{3}$ and $\mathrm{KBiO}_{3}$. The experimental determination of the band gaps available in the literature for $\mathrm{NaBiO}_{3}$ and $\mathrm{KBiO}_{3}$ are based on UV-vis diffused-reflectance spectra [39,89]. This returns us a value of $2.6 \mathrm{eV}$ and $2.1 \mathrm{eV}$, respectively, for $\mathrm{NaBiO}_{3}$ and $\mathrm{KBiO}_{3}$, which are consistent with our data considering the systematic GGA underestimation of the band gaps. The GLLB-SC description of the band gap seems to improve the results, although our theoretical estimate remains smaller than the experimental observation.

Note that the role of the alkali metals is minor but not negligible. In general, we find a systematic band gap increase as the ionic radius of the alkali metal gets larger. This appears to be related to the general increase in cell volume and hence to the weakening of the orbital hybridization. Enforcing this argument is the bandwidth reduction when going from $\mathrm{Na}$ - to $\mathrm{K}$-based compounds, which is, for instance, particularly evident in the conduction bands of $\mathrm{NaBiO}_{2}$ and $\mathrm{KBiO}_{2}$. Finally, we observe that $\mathrm{SOC}$ has little effect on $\mathrm{ABiO}_{3}$ compounds. Both the CBM and VBM are almost unaffected by SOC, as expected from their $\mathrm{O}-p$ character, and the only notable changes in the band structure (in the energy range considered in Fig. 3) are in the valence manifold for energies lower than $2 \mathrm{eV}$ from the VBM. This is the only energy region where the PDOS shows a non-negligible Bi- $p$ contribution. Let us now turn our attention to the band structures of the sulphides, again, starting from the $A \mathrm{BiS}_{2}$ composition. Broadly speaking, $\mathrm{NaBiS}_{2}$ (Fig. 4) and $\mathrm{KBiS}_{2}$ (Fig. S5 of the SM [71]) have an electronic structure similar to that of their oxide counterparts, namely they present a valence band with a strong S- $p$

TABLE III. Energy gaps calculated using the Kohn-Sham spectrum obtained with PBE ( $\left.\Delta E_{\mathrm{PBE}}\right)$, GLLB-SC ( $\left.\Delta E_{\mathrm{GLLBsc}}\right)$, and extracted from the imaginary part of the RPA-PBE dielectric function (optical gap) $\Delta E_{\text {opt }}$ are reported. For comparison, we report both the direct and indirect gaps calculated using the $\mathrm{PBE}$ functional. $E_{\mathrm{R}}$ is the experimentally reported band gap. Two compounds, namely $\mathrm{NaBiO}_{2}$ and $\mathrm{KBiO}_{2}$, are direct band gap materials, while all the others are indirect. Note that the band gaps reported from literature for $\mathrm{KBiO}_{3}, \mathrm{NaBiS}_{2}$, and $\mathrm{KBiS}_{2}$ are always associated to the cubic structure, while our results are for their trigonal phase (see discussion). Also, the reported energy gaps, $E_{\mathrm{R}}$, for $\mathrm{NaBiO}_{2}$ and $\mathrm{KBiO}_{2}$ are theoretical values (no experimental determination exists). In the last two columns, we report the average static dielectric function $\bar{\varepsilon}$ and its variance $\Delta \varepsilon$ (see definition in the main text).

Energy gaps (eV)

\begin{tabular}{|c|c|c|c|c|c|c|c|}
\hline \multirow[b]{2}{*}{ Compound } & \multicolumn{2}{|c|}{$E_{\mathrm{PBE}}$} & \multirow[b]{2}{*}{$E_{\text {GLLB-SC }}$} & \multirow[b]{2}{*}{$E_{\mathrm{opt}}$} & \multirow[b]{2}{*}{$E_{\mathrm{R}}$} & \multirow[b]{2}{*}{$\bar{\varepsilon}$} & \multirow[b]{2}{*}{$\Delta \varepsilon$} \\
\hline & Direct & $\overline{\text { Indirect }}$ & & & & & \\
\hline $\mathrm{NaBiO}_{2}$ & 1.35 & & 1.93 & 1.42 & $1.60[33]$ & 2.35 & 0.68 \\
\hline $\mathrm{NaBiO}_{3}$ & 1.16 & 1.02 & 1.80 & 1.33 & 2.60 [89] & 2.58 & 0.09 \\
\hline $\mathrm{KBiO}_{2}$ & 1.80 & & 2.47 & 1.89 & $2.00[34]$ & 2.16 & 0.66 \\
\hline $\mathrm{KBiO}_{3}$ & 1.25 & 1.17 & 1.82 & 1.47 & 2.10 [39] & 2.36 & 0.23 \\
\hline $\mathrm{NaBiS}_{2}$ & 2.10 & 1.51 & 2.03 & 2.19 & $0.44-1.41[41,60,92,93]$ & 5.79 & 0.02 \\
\hline $\mathrm{NaBiS}_{3}$ & 0.89 & 0.80 & 1.24 & 0.90 & & 3.82 & 1.90 \\
\hline $\mathrm{KBiS}_{2}$ & 2.19 & 1.64 & 2.15 & 2.14 & $0.90,1.61[41,93]$ & 5.31 & 0.06 \\
\hline $\mathrm{KBiS}_{3}$ & 1.44 & 1.26 & 1.79 & 1.49 & & 4.58 & 1.84 \\
\hline
\end{tabular}




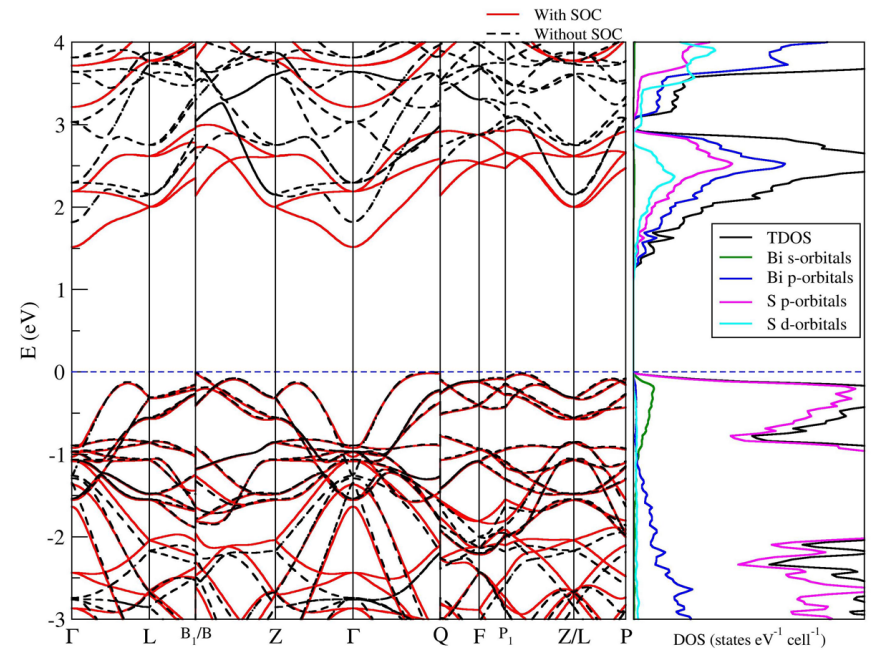

FIG. 4. The calculated DFT energy band structure and PDOS of $\mathrm{NaBiS}_{2}$. The compound is an indirect band-gap semiconductor with its VBM at $\mathrm{Q}$ and the $\mathrm{CBM}$ at $\Gamma$. The red and the dashed-black lines represent, respectively, the bands calculated with and without including spin-orbit coupling (SOC). The PDOS on the right-hand side is for the band structure calculated with SOC.

character and hybridization with the Bi- $s$ and Bi- $p$ shells at its top. The dispersion is significantly less pronounced than in the oxide case owning a further unit cell volume increase. Both $\mathrm{NaBiS}_{2}$ and $\mathrm{KBiS}_{2}$ are indirect band-gap semiconductors with the CBM at $\Gamma$. The position of the VBM is at $\mathrm{Q}$ for $\mathrm{NaBiS}_{2}$ and at a low-symmetry point along the Z-to- $\Gamma$ direction for $\mathrm{KBiS}_{2}$. Notably for $\mathrm{KBiS}_{2}$, the VBM is almost degenerate with several other minima extremely close in energy and all located at low symmetry points in the Brillouin zone (e.g., $\Gamma$ to $\mathrm{Q}, \Gamma$ to $\mathrm{L}$ ).

The inclusion of SOC has a much more pronounced effect than in the case of the oxides. In fact, there is a split of the conduction band into a lower and an upper manifold with a net reduction of the band gap with respect to that calculated without considering spin orbit. Such drastic reduction is similar to what was found for $\mathrm{Pb}$-based halide perovskites [90,91], which share with the compounds investigated here the octahedral local coordination of the heavy metal $(\mathrm{Pb}$ and $\mathrm{Bi})$. In contrast, the local coordination of $\mathrm{Bi}$ in $A \mathrm{BiO}_{2}$ is quasitetrahedral, and this seems to be the main difference in the behavior of the sulphides and oxides against SOC. In any case, for both $\mathrm{NaBiS}_{2}$ and $\mathrm{KBiS}_{2}$, the band gap remains larger than that of their corresponding oxides. There are a number of experimental determinations of the band gap of $\mathrm{NaBiS}_{2}$ and $\mathrm{KBiS}_{2}$ (see Table III). These seem to return the measured band gaps rather narrow and constantly smaller than those computed with the GGA and the GLLB-SC functional. Such a result is somehow unexpected, given the consistent GGA underestimation of the band gaps and the typical good description given by GLLB-SC. However, it has to be noticed that in all the experimental works $[60,92,93]$ the lattice structure is reported cubic, although no further crystallographic characterization is provided. Our calculated band gap are for the trigonal structure. As speculated before, we believe that the experimental structure is actually a macro-

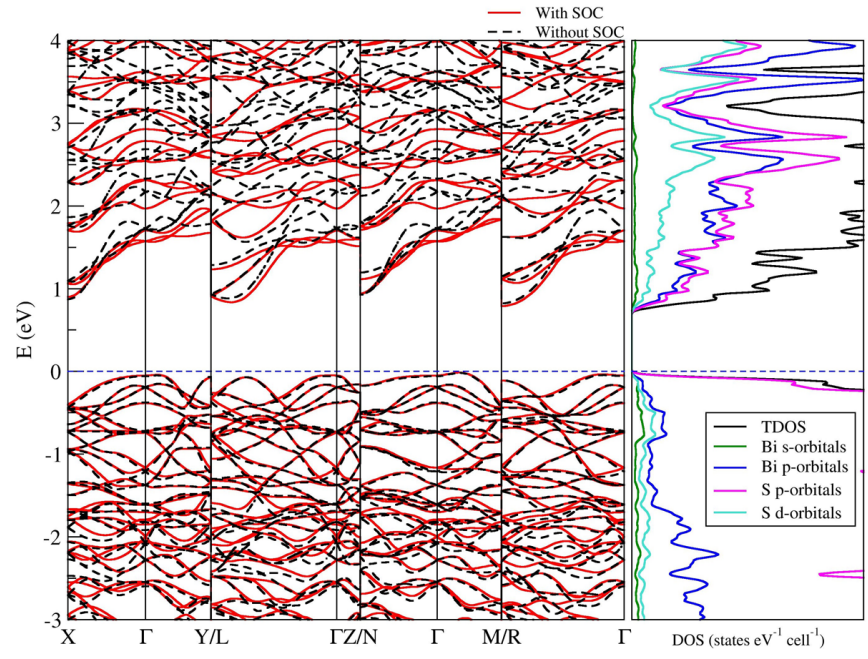

FIG. 5. The calculated DFT energy band structure and PDOS of $\mathrm{NaBiS}_{3}$. The compound is an indirect band-gap semiconductor with its VBM lying between $\Gamma$ and $\mathrm{M}$ and the CBM at R. The red and the dashed-black lines represent, respectively, the bands calculated with and without including spin-orbit coupling (SOC). The PDOS on the right-hand side is for the band structure calculated with SOC.

scopic average of local trigonal lattices. Hence, the measured band gap is the result of such average, which needs to be taken over the crystal structure orientation and the site occupation. We then expect that the actual band gap will be significantly smaller than that computed for a homogeneous trigonal lattice with an ordered site-occupation configuration. As a further test we have computed the band gaps of the metastable cubic structures of $\mathrm{NaBiS}_{2}$ and $\mathrm{KBiS}_{2}$. PBE returns values of $1.46 \mathrm{eV}$ and $1.56 \mathrm{eV}$ for $\mathrm{NaBiS}_{2}$ (lattice constant $5.76 \AA$ ) and $\mathrm{KBiS}_{2}$ (lattice constant $5.95 \AA$ ), respectively. These are rather similar to those obtained for the trigonal phases and again systematically larger than what measured. Such discrepancy between predictions and experiments appears to corroborate further the idea that actual samples are not in a single cubic phase, but they are rather a macroscopic average of equivalent trigonal ones.

Finally, we look at $\mathrm{NaBiS}_{3}$ (Fig. 5) and $\mathrm{KBiS}_{3}$ (Fig. S6 of the SM [71]). Their PDOSs have some similarities with those computed for the oxides with the same stoichiometry, in particular, for what concerns the top of the valence band, which is completely dominated by $\mathrm{O}-p$ states. The conduction band instead appears to present a strong mixture between $\mathrm{O}-p$ and $\mathrm{Bi}-p$ contributions, consistent with the nominal $\mathrm{Bi}^{+5}$ valence configuration. This is different from what was found for $A \mathrm{BiO}_{3}$, in which the $\mathrm{O}$ and $\mathrm{Bi}$ contributions to the conduction band are well separated. Such difference is a consequence of both the lower electron affinity of $\mathrm{S}$ with respect to $\mathrm{O}$ and of the different local Bi coordination in oxides and sulphides. Also, notable is the presence of a $\mathrm{Bi}-d$ orbital contribution across the entire valence manifold.

Both $\mathrm{NaBiS}_{3}$ and $\mathrm{KBiS}_{3}$ are predicted to be indirect gap semiconductors. $\mathrm{NaBiS}_{3}$ has the VBM at a low-symmetry point along the $\Gamma$-to-M direction and the $\mathrm{CBM}$ at $\mathrm{R}$, while for $\mathrm{KBiS}_{3}$ the VBM is $\mathrm{M}$ and the CBM is at X. In both cases, there are other points in the Brillouin zone with energy close 


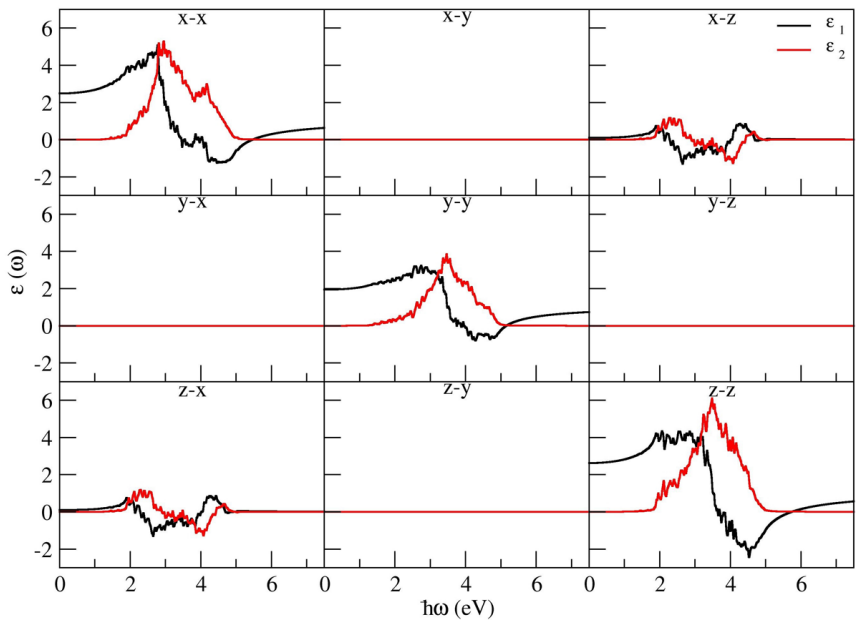

FIG. 6. Real, $\varepsilon_{1}(\omega)$ (black line), and imaginary, $\varepsilon_{2}(\omega)$ (red line), component of the dielectric tensor of $\mathrm{NaBiO}_{2}$ as a function of energy.

to that of the band extremal points owning to the relatively low dispersion. The PBE (GLLB-SC) gap values are $0.89 \mathrm{eV}$ $(1.24 \mathrm{eV})$ and $1.26 \mathrm{eV}(1.79 \mathrm{eV})$, respectively, for $\mathrm{NaBiS}_{3}$ and $\mathrm{KBiS}_{3}$. As expected, they are smaller than those of the corresponding oxides and, as in the oxides case, the largest gap is associated to the alkaline metal presenting the largest ionic radius $\mathrm{K}$. We are not aware of any experimental or theoretical determination of the gap, so that our results remain the only available. Finally, we observe that SOC has almost no effect on the top of the valence band, which is primarily dominated by S- $p$ states, while it distorts the lower part of the conduction one, where the Bi content to the PDOS is larger. Notably, such distortion is not associated to a significant alteration of the size of the band gap.

\section{E. Dielectric properties}

The interband contribution to the linear dielectric tensor calculated within the RPA over the PBE band structure for all the eight compounds are now discussed. The calculations were carried out for an energy range between $0 \mathrm{eV}$ and $7.5 \mathrm{eV}$ in order to include the infrared, visible, and ultraviolet region of the electromagnetic spectra. In particular, for all compounds we compute both the real $\varepsilon_{1}(\omega)$ and imaginary $\varepsilon_{2}(\omega)$ part of the dielectric function and we define the optical gap at the minimum onset of the three diagonal components of $\varepsilon_{2}(\omega)$. Let us start our analysis from $\mathrm{NaBiO}_{2}$ and $\mathrm{KBiO}_{2}$, whose dielectric functions are displayed in Fig. 6 and Fig. S7 of the SM [71], respectively. As expected from the rather similar electronic structure, $\mathrm{NaBiO}_{2}$ and $\mathrm{KBiO}_{2}$ have very similar dielectric functions. The diagonal component of the dielectric tensors are relatively anisotropic, as expected from the low symmetry lattice, and the only non-negligible offdiagonal component is the $x z$ one (identical to the $z x$ one since $\varepsilon$ is a symmetric tensor). The optical band gap is found for both $\mathrm{NaBiO}_{2}$ and $\mathrm{KBiO}_{2}$ in the $y y$ component and it coincides with the direct Kohn-Sham band gap at $\Gamma$ (see Table III). This means that the direct transition from the VBM to the CBM is allowed. Note that the optical gap does not exactly

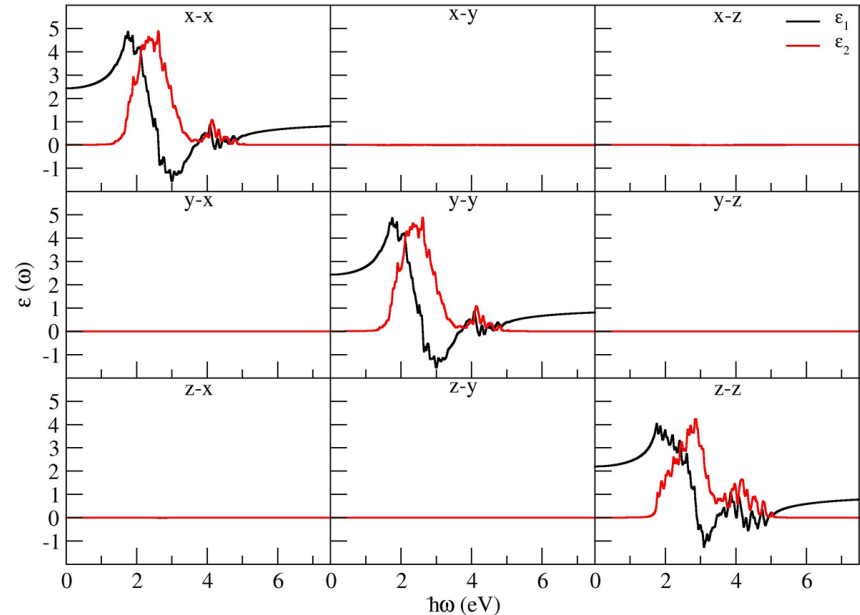

FIG. 7. Real, $\varepsilon_{1}(\omega)$ (black line), and imaginary, $\varepsilon_{2}(\omega)$ (red line), component of the dielectric tensor of $\mathrm{NaBiO}_{3}$ as a function of energy.

coincide with the Kohn-Sham one, because of the broadening introduced when computing the dielectric function.

The dielectric functions of the $A \mathrm{BiO}_{3}$ compounds are presented in Fig. $7\left(\mathrm{NaBiO}_{3}\right)$ and Fig. S8 of the $\mathrm{SM}\left(\mathrm{KBiO}_{3}[71]\right)$. In this case, the dielectric tensor is rather isotropic and diagonal, owning to the high symmetry of the crystal structure. For both $\mathrm{NaBiO}_{3}$ and $\mathrm{KBiO}_{3}$, we find that the optical gap is about $0.2 \mathrm{eV}$ larger than the direct Kohn-Sham one (PBE), indicating that transitions at the band edges are forbidden, or at least highly suppressed (the lowest among the conduction bands has high dispersion, so that the density of transitions is low). In fact, the onset of $\varepsilon_{2}(\omega)$ corresponds to excitations from the VBM to the second of the conduction bands.

Moving to the sulphides, the dielectric constant profiles are shown in Figs. S7 through S12 of the SM [71]. In all cases, the tensor is nondiagonal and generally anisotropic. Such anisotropy appears genuine for the $A \mathrm{BiS}_{3}$ compounds, while for the $A \mathrm{BiS}_{2}$ we have to notice, once again, that the results reported here correspond to a specific arrangement of the $\mathrm{Bi}$ and $\mathrm{Na} / \mathrm{K}$ ions. Thus, it is reasonable to expect that such anisotropy will be strongly suppressed, once the appropriate macroscopic average over the atomic distribution and distortion is taken. The optical band gaps of $\mathrm{NaBiS}_{2}$ and $\mathrm{KBiS}_{2}$ are generally larger than the Kohn-Sham ones by about $0.5 \mathrm{eV}$ and correspond to the first available direct transitions, namely the optical gap is simply the Kohn-Sham direct gap. As noted before, these band gaps are larger than those reported in the literature (see discussion in the previous section). Finally, for $\mathrm{NaBiS}_{3}$ and $\mathrm{KBiS}_{3}$, the optical band gap are only marginally larger than the Kohn-Sham one and again correspond to the first available direct transition.

To conclude this section we discuss the static dielectric constant. The average value of its diagonal components, $\bar{\varepsilon}$, and the corresponding variance, $\Delta \varepsilon=\left|\max _{\alpha} \varepsilon_{\alpha \alpha}-\min _{\alpha} \varepsilon_{\alpha \alpha}\right|$, are reported in the last two columns of Table III, while detailed information about the full static dielectric tensor is included in the SM [71]. In general, we find the sulphides to have an average dielectric constant larger than that of the oxides, with Na-based sulphide presenting the largest values. Furthermore, as expected, we find that the asymmetry of the dielectric 
constant, as measured via $\Delta \varepsilon$, follows the symmetry of the associated lattice structure. The triclinic $\mathrm{ABiS}_{3}$ and monoclinic $A_{B i O}$ compounds present the largest variations, while for the remaining trigonal structures the diagonal elements of the static dielectric tensor are rather similar to each other. This trend follows closely to that found for the dynamic dielectric functions.

\section{CONCLUSIONS}

Motivated by the need for finding a $\mathrm{Pb}$-free replacement of $\mathrm{MAPbI}_{3}$ as light-harvesting media, we have presented an extensive computational study of the structural, thermodynamic, elastic, electronic, and dielectric properties of alkalimetal-based bismuth ternary chalcogenides. The structures of the various compounds have been calculated in agreement with available crystallographic data, with the exception of $\mathrm{NaBiS}_{2}$ and $\mathrm{KBiS}_{2}$, for which DFT has returned us a trigonal instead of a cubic structure. Consistency between theory and experiments is then reached by assuming that the experimental samples, known to have the site-occupation disorder, are a macroscopic average of the various equivalent trigonal structures.

We have found that all the compounds investigated are thermodynamically and mechanically stable. An analysis of their elastic properties has revealed that the bulk, shear, and Young's moduli get smaller as the crystal structure goes from trigonal to monoclinic and finally to triclinic. These compounds present a semiconducting band structure with DFT GGA gaps ranging between $0.80 \mathrm{eV}$ and $1.80 \mathrm{eV}$. The optical gaps are marginally larger than the quasiparticle ones owing to the fact that they are indirect. Notably, in all cases the difference between the direct and indirect gap is not large, since the extremal bands are relatively flat. $\mathrm{NaBiO}_{2}$ and $\mathrm{KBiO}_{2}$ have a direct gap. Considering the well-known tendency of the GGA to underestimate the quasiparticle gap, we expect these compounds to absorb light in the early blue region of the electromagnetic spectrum. On the grounds of their thermodynamic stability, electronic, and optical properties, we foresee these materials as alternative lead-free visible-light absorbing semiconducting materials. In all compounds, the conduction band is dominated by Bi-p orbitals as suggested by the $\mathrm{Bi}^{3+}$ and $\mathrm{Bi}^{5+}$ nominal oxidation state. This is similar to what was found for lead-based halides hybrid perovskites, and we expect for the $\mathrm{Bi}$-based compounds the exciton binding energy to be small. $\mathrm{NaBiO}_{3}$ and $\mathrm{KBiO}_{3}$ are the exceptions, since the band gap is between $\mathrm{O}-p$ orbitals. A small exciton binding energy together with an indirect gap make these compounds interesting [94] as potential media for photocatalysis and water splitting. This is an area that certainly deserves further study, which is currently ongoing.

\section{ACKNOWLEDGMENTS}

S.K.R. thanks Colibaba Anais Stefana, Rui Dong, Carlo Motta, and Florian Knoop for the useful discussions. This work is supported by Institute of Technology Sligo President's Bursary Award under the Grant No. PPRES050. S.S. thanks the Irish Research Council (IRCLA/2019/127) for financial support. F.E. thanks the Qatar National Research Fund (QNRF) through the National Priorities Research Program (NPRP8-090-2-047). Computational resources have been provided by the Trinity Centre for High Performance Computing (TCHPC) (Project Code: HPC_16_00953) and Irish Centre for HighEnd Computing (ICHEC) (Project Codes: isphy001c and isphy002c).
[1] D. Sica, O. Malandrino, S. Supino, M. Testa, and M. C. Lucchetti, Renew. Sust. Energ. Rev. 82, 2934 (2018).

[2] M. Tao, Terawatt Solar Photovoltaics (Springer, London, 2014), pp. 9-20.

[3] D. Ginley, M. A. Green, and R. Collins, MRS Bull. 33, 355 (2008).

[4] C. Deibel and V. Dyakonov, Rep. Prog. Phys. 73, 096401 (2010).

[5] A. Hagfeldt, G. Boschloo, L. Sun, L. Kloo, and H. Pettersson, Chem. Rev. 110, 6595 (2010).

[6] M. Yamaguchi, Sol. Energy Mater. Sol. Cells 75, 261 (2003).

[7] T. M. Brenner, D. A. Egger, L. Kronik, G. Hodes, and D. Cahen, Nat. Rev. Mater. 1, 15007 (2016).

[8] M. Wolf, Proc. IREE 48, 1246 (1960).

[9] A. Poglitsch and D. Weber, J. Chem. Phys. 87, 6373 (1987).

[10] D. Mitzi, C. Feild, W. Harrison, and A. Guloy, Nature (London) 369, 467 (1994).

[11] H.-S. Kim, C.-R. Lee, J.-H. Im, K.-B. Lee, T. Moehl, A. Marchioro, S.-J. Moon, R. Humphry-Baker, J.-H. Yum, J. E. Moser et al., Sci. Rep. 2, 591 (2012).

[12] M. A. Green, E. D. Dunlop, J. Hohl-Ebinger, M. Yoshita, N. Kopidakis, and A. W. Y. Ho-Baillie, Progress in Photovoltaics: Research and Applications 28, 3 (2019).
[13] M. M. Lee, J. Teuscher, T. Miyasaka, T. N. Murakami, and H. J. Snaith, Science 338, 643 (2012).

[14] H. Zhou, Q. Chen, G. Li, S. Luo, T.-b. Song, H.-S. Duan, Z. Hong, J. You, Y. Liu, and Y. Yang, Science 345, 542 (2014).

[15] T. Salim, S. Sun, Y. Abe, A. Krishna, A. C. Grimsdale, and Y. M. Lam, J. Mater. Chem. A 3, 8943 (2015).

[16] Y. Zhao and K. Zhu, J. Phys. Chem. Lett. 5, 4175 (2014).

[17] T.-B. Song, Q. Chen, H. Zhou, C. Jiang, H.-H. Wang, Y. M. Yang, Y. Liu, J. You, and Y. Yang, J. Mater. Chem. A 3, 9032 (2015).

[18] S. De Wolf, J. Holovsky, S.-J. Moon, P. Lolper, B. Niesen, M. Ledinsky, F.-J. Haug, J.-H. Yum, and C. Ballif, J. Phys. Chem. Lett. 5, 1035 (2014).

[19] Y.-Y. Zhang, S. Chen, P. Xu, H. Xiang, X.-G. Gong, A. Walsh, and S.-H. Wei, Chin. Phys. Lett. 35, 036104 (2018).

[20] D. P. McMeekin, G. Sadoughi, W. Rehman, G. E. Eperon, M. Saliba, M. T. Hörantner, A. Haghighirad, N. Sakai, L. Korte, B. Rech et al., Science 351, 151 (2016).

[21] N. K. Noel, S. D. Stranks, A. Abate, C. Wehrenfennig, S. Guarnera, A.-A. Haghighirad, A. Sadhanala, G. E. Eperon, S. K. Pathak, M. B. Johnston et al., Energy Environ. Sci. 7, 3061 (2014). 
[22] C. N. Savory, A. Walsh, and D. O. Scanlon, ACS Energy Lett. 1, 949 (2016).

[23] W.-J. Yin, J.-H. Yang, J. Kang, Y. Yan, and S.-H. Wei, J. Mater. Chem. A 3, 8926 (2015).

[24] M. Lyu, J.-H. Yun, M. Cai, Y. Jiao, P. V. Bernhardt, M. Zhang, Q. Wang, A. Du, H. Wang, G. Liu et al., Nano Research 9, 692 (2016).

[25] X. Gonze, J.-P. Michenaud, and J.-P. Vigneron, Phys. Scr. 37, 785 (1988).

[26] F. Vines, M. Bernechea, G. Konstantatos, and F. Illas, Phys. Rev. B 94, 235203 (2016).

[27] B. Ghosh, B. Wu, H. K. Mulmudi, C. Guet, K. Weber, T. C. Sum, S. Mhaisalkar, and N. Mathews, ACS Appl. Mater. Interfaces 10, 35000 (2018).

[28] R. L. Hoye, L. C. Lee, R. C. Kurchin, T. N. Huq, K. H. Zhang, M. Sponseller, L. Nienhaus, R. E. Brandt, J. Jean, J. A. Polizzotti et al., Adv. Mater. 29, 1702176 (2017).

[29] F. Demartin, C. Gramaccioli, and I. Campostrini, Mineral. Mag. 74, 141 (2010).

[30] L. C. Lee, T. N. Huq, J. L. MacManus-Driscoll, and R. L. Hoye, APL Mater. 6, 084502 (2018).

[31] B.-W. Park, B. Philippe, X. Zhang, H. Rensmo, G. Boschloo, and E. M. Johansson, Adv. Mater. 27, 6806 (2015).

[32] S. Öz, J.-C. Hebig, E. Jung, T. Singh, A. Lepcha, S. Olthof, F. Jan, Y. Gao, R. German, P. H. van Loosdrecht et al., Sol. Energy Mater. Sol. Cells 158, 195 (2016).

[33] E. Keller and C. Röhr, Zeit. Krist.-Crystalline Materials 223, 431 (2008).

[34] N. Zoche and M. Jansen, Z. Anorg. Allg. Chem. 624, 205 (1998).

[35] N. Kumada, N. Kinomura, and A. Sleight, Mater. Res. Bull. 35, 2397 (2000).

[36] J. Liu, S. Chen, Q. Liu, Y. Zhu, and J. Zhang, Chem. Phys. Lett. 572, 101 (2013).

[37] S. Chen, Z. Cao, and X. Fu, Mater. Chem. Phys. 142, 748 (2013).

[38] L. Cheng and Y. Kang, Mater. Lett. 117, 94 (2014).

[39] R. Ramachandran, M. Sathiya, K. Ramesha, A. Prakash, G. Madras, and A. Shukla, J. Chem. Sci. 123, 517 (2011).

[40] J. Boon, Recl. Trav. Chim. 63, 32 (1944).

[41] B. Gabrel'yan, A. Lavrentiev, I. Y. Nikiforov, and V. Sobolev, J. Struct. Chem. 49, 788 (2008).

[42] V. Blum, R. Gehrke, F. Hanke, P. Havu, V. Havu, X. Ren, K. Reuter, and M. Scheffler, Comput. Phys. Commun. 180, 2175 (2009).

[43] J. P. Perdew, M. Ernzerhof, and K. Burke, J. Chem. Phys. 105, 9982 (1996).

[44] J. D. Head and M. C. Zerner, Chem. Phys. Lett. 122, 264 (1985).

[45] T. Bučko, S. Lebègue, J. Hafner, and J. G. Angyan, Phys. Rev. B 87, 064110 (2013).

[46] G.-X. Zhang, A. Tkatchenko, J. Paier, H. Appel, and M. Scheffler, Phys. Rev. Lett. 107, 245501 (2011).

[47] C. Motta, F. El-Mellouhi, S. Kais, N. Tabet, F. Alharbi, and S. Sanvito, Nat. Commun. 6, 7026 (2015).

[48] A. Tkatchenko and M. Scheffler, Phys. Rev. Lett. 102, 073005 (2009).

[49] C. Ambrosch-Draxl and J. O. Sofo, Comput. Phys. Commun. 175, 1 (2006).

[50] G. Kresse and J. Furthmüller, Phys. Rev. B 54, 11169 (1996).
[51] P. E. Blöchl, Phys. Rev. B 50, 17953 (1994).

[52] O. Gritsenko, R. van Leeuwen, E. van Lenthe, and E. J. Baerends, Phys. Rev. A 51, 1944 (1995).

[53] J. J. Mortensen, L. B. Hansen, and K. W. Jacobsen, Phys. Rev. B 71, 035109 (2005).

[54] M. Kuisma, J. Ojanen, J. Enkovaara, and T. T. Rantala, Phys. Rev. B 82, 115106 (2010).

[55] I. E. Castelli, T. Olsen, S. Datta, D. D. Landis, S. Dahl, K. S. Thygesen, and K. W. Jacobsen, Energy Environ. Sci. 5, 5814 (2012).

[56] G. Pilania, A. Mannodi-Kanakkithodi, B. Uberuaga, R. Ramprasad, J. Gubernatis, and T. Lookman, Sci. Rep. 6, 19375 (2016).

[57] A. Togo and I. Tanaka, Scr. Mater. 108, 1 (2015).

[58] A. Jain, S. Ong, G. Hautier, W. Chen, W. Richards, S. Dacek, S. Cholia, D. Gunter, D. Skinner, G. Ceder, and K. Persson, APL Mat. 1, 011002 (2013).

[59] O. Glemser and M. Filcek, Z. Anorg. Allg. Chem. 279, 321 (1955).

[60] B. A. Rosales, M. A. White, and J. Vela, J. Am. Chem. Soc. 140, 3736 (2018).

[61] S. Curtarolo, W. Setyawan, S. Wang, J. Xue, K. Yang, R. H. Taylor, L. J. Nelson, G. L. W. Hart, S. Sanvito, M. BuongiornoNardelli, N. Mingo, and O. Levy, Comput. Mater. Sci. 58, 275 (2012).

[62] C. Oses, E. Gossett, D. Hicks, F. Rose, M. Mehl, E. Perim, I. Takeuchi, S. Sanvito, M. Scheffler, Y. Lederer, O. Levy, C. Toher, and S. Curtarolo, J. Chem. Inf. Model. 58, 2477 (2018).

[63] W. Setyawan and S. Curtarolo, Comput. Mater. Sci. 49, 299 (2010).

[64] B. Schwedes and R. Hoppe, Z. Anorg. Allg. Chem. 392, 97 (1972).

[65] N. Kumada, N. Kinomura, P. Woodward, and A. Sleight, J. Solid State Chem. 116, 281 (1995).

[66] H. Park, F. Alharbi, S. Sanvito, N. Tabet, and F. El-Mellouhi, ChemPhysChem 19, 703 (2018).

[67] H. Park, F. H. Alharbi, S. Sanvito, N. Tabet, and F. El-Mellouhi, J. Phys. Chem. C 122, 8814 (2018).

[68] J. W. Bennett, I. Grinberg, and A. M. Rappe, Phys. Rev. B 79, 235115 (2009).

[69] M.-K. Han, Y.-S. Jin, B. Yu, W. Choi, T.-S. You, and S. Kim, J. Mat. Chem. A 4, 13859 (2016).

[70] X.-G. Zhao, G. M. Dalpian, Z. Wang, and A. Zunger, Phys. Rev. B 101, 155137 (2020).

[71] See Supplemental Material at http://link.aps.org/supplemental/ 10.1103/PhysRevMaterials.4.075401 for the calculated elastic stiffness constants of the eight alkali-metal based bismuth chalcogenides, the calculated phonon spectra of $\mathrm{NaBiS}_{2}$ and $\mathrm{KBiS}_{2}$, the calculated DFT energy band structure and PDOS of $\mathrm{KBiO}_{2}, \mathrm{KBiO}_{3}, \mathrm{KBiS}_{2}$, and $\mathrm{KBiS}_{3}$, the variation in the real and imaginary parts of the dielectric function of $\mathrm{KBiO}_{2}$, $\mathrm{KBiO}_{3}, \mathrm{NaBiS}_{2}, \mathrm{NaBiS}_{3}, \mathrm{KBiS}_{2}$, and $\mathrm{KBiS}_{3}$, and the detailed information about the static dielectric tensor of the alkali-metalbased bismuth ternary chalcogenides.

[72] S. Sanvito, C. Oses, J. Xue, A. Tiwari, M. Zic, T. Archer, P. Tozman, M. Venkatesan, J. Coey, and S. Curtarolo, Sci. Adv. 3, e1602241 (2017).

[73] C. Toher, C. Oses, D. Hicks, and S. Curtarolo, npj Comp. Mater. 5, 69 (2019). 
[74] S. Ong, W. Richards, A. Jain, G. Hautier, M. Kocher, S. Cholia, D. Gunter, V. Chevrier, K. Persson, and G. Ceder, Comput. Mater. Sci. 68, 314 (2013).

[75] S. Ong, L. Wang, B. Kang, and G. Ceder, Chem. Mater. 20, 1798 (2008).

[76] S. Ong, A. Jain, G. Hautier, B. Kang, and G. Ceder, Electrochem. Commun. 12, 427 (2010).

[77] K. H. Whitmire, Bismuth: Inorganic chemistry, Encyclopedia of Inorganic and Bioinorganic Chemistry (American Cancer Society, 2014), pp. 1-32.

[78] M. Born and K. Huang, Dynamical theory of crystal lattices (Clarendon Press, Oxford, England, 1954).

[79] B. Karki, G. Ackland, and J. Crain, J. Phys.: Condens. Matter 9, 8579 (1997).

[80] F. Mouhat and F.-X. Coudert, Phys. Rev. B 90, 224104 (2014).

[81] Q.-J. Liu, Z.-T. Liu, and L.-P. Feng, Phys. Status Solidi B 248, 1629 (2011).

[82] N. BD, J. Phys. Soc. Jpn. 20, 635 (1965).

[83] Y.-C. Zhou, C. Zhao, F. Wang, Y.-J. Sun, L.-Y. Zheng, and X.-H. Wang, J. Am. Ceram. Soc. 96, 3891 (2013).
[84] Z.-j. Wu, E.-j. Zhao, H.-p. Xiang, X.-f. Hao, X.-j. Liu, and J. Meng, Phys. Rev. B 76, 054115 (2007).

[85] R. Hill, Proc. Phys. Soc., Sect. A 65, 349 (1952).

[86] H. Koc, A. M. Mamedov, E. Deligoz, and H. Ozisik, arXiv:1205.3344.

[87] S. Chen, Y. Sun, Y.-H. Duan, B. Huang, and M.-J. Peng, J. Alloys Compd. 630, 202 (2015).

[88] S. Pugh, Lond. Edinb. Dubl. Phil. Mag. 45, 823 (1954).

[89] T. Kako, Z. Zou, M. Katagiri, and J. Ye, Chem. Mater. 19, 198 (2007).

[90] P. Umari, E. Mosconi, and F. D. Angelis, Sci. Rep. 4, 4467 (2014).

[91] J. Even, L. Pedesseau, J.-M. Jancu, and C. Katan, J. Phys. Chem. Lett. 4, 2999 (2013).

[92] S. Kang, Y. Hong, and Y. Jeon, Bull. Korean Chem. Soc. 35, 1887 (2014).

[93] C. Yang, Z. Wang, Y. Wu, Y. Lv, B. Zhou, and W.-H. Zhang, ACS Appl. Energy Mater. 2, 182 (2019).

[94] X. Lv, W. Wei, Q. Sun, F. Li, B. Huang, and Y. Dai, Appl. Catal., B 217, 275 (2017). 